\title{
Concepciones de estudiantes sobre enseñanza socialmente justa. Un estudio fenomenográfico
}

\author{
Students Conceptions on Socially Just Teaching. A \\ Phenomenographic Study
}

\author{
F. Javier Murillo \\ Nina Hidalgo \\ Universidad Autónoma de Madrid
}

Recibido: $14 / 10 / 2018$

Aceptado: 17/12/2018

\begin{abstract}
School has a fundamental role in the fight against social injustices. In particular, instruction practice and how they develop teaching is a determining element in the achievement of a more just society. The present investigation focuses on knowing the conceptions of students about what a socially just teaching is like. To achieve this, a phenomenological study was carried out with 32 students of Primary Education and Secondary Education of the Community of Madrid. The results show that the students consider that socially just teaching is one that: a) favors the integral development of students, b) works content sensitive to social injustices, c) promotes the participation of students in the process of learning, d) poses a useful and autonomous learning and e) teaches students to think and be critical.
\end{abstract}

KEY WORDS: Socially just teaching, Student conceptions; Phenomenography, Education for Social Justice.

\section{RESUMEN}

La escuela tiene un papel fundamental en la lucha contra las injusticias sociales. En concreto, la práctica docente y cómo desarrollen la enseñanza los y las profesores es un elemento determinante en la consecución de una sociedad más justa. El presente estudio se centra en conocer las concepciones de estudiantes acerca de cómo es una enseñanza socialmente justa. Para lograrlo, se ha llevado a cabo un estudio fenomenográfico con 32 estudiantes de Educación Primaria y de Educación Secundaria de la Comunidad de Madrid. Los resultados muestran que los y las estudiantes consideran que enseñanza socialmente justa es aquella que: a) favorece el desarrollo integral del alumnado, b) trabaja contenidos sensibles a las injusticias sociales, c) promueve la participación de los y las estudiantes en el proceso de aprendizaje, d) plantea un aprendizaje útil y autónomo y e) enseña al alumnado a pensar y a ser críticos.

PALABRAS CLAVE: Enseñanza socialmente justa, Concepciones de estudiantes; Fenomenografía, Educación para la Justicia Social. 


\section{Introducción}

¿Puede la educación cambiar la sociedad o solo es un medio de reproducción de las desigualdades sociales? La respuesta, como recuerda Apple (2012), es mucho más compleja y llena de matices de lo que se podría suponer. Aunque, en esencia, podríamos decir que depende de lo que hagan las escuelas: algunas, por acción u omisión, contribuyen al mantenimiento de las desigualdades, mientras que otras realizan aportaciones significativas a la promoción de la Justicia Social (Griffiths, 2003; Kumashiro, 2015; Murillo y Hernández-Castilla, 2011). La clave es lo que la escuela haga y piense: los procesos de enseñanza y de aprendizaje, la organización del centro y la cultura del centro.

Incluso se podría afirmar que sólo cuando el centro educativo hace explícita su intención de luchar contra las desigualdades e injusticias de la sociedad podrá lograrlo (Murillo y HernándezCastilla, 2014). Si la escuela no se plantea esta lucha activa contra las desigualdades, estará reproduciendo y manteniendo las desigualdades en la sociedad, convirtiéndose en un mecanismo del sistema para mantener las diferencias sociales.

De esta forma, existe una creciente literatura, especialmente anglosajona, sobre "Enseñanza para la Justicia Social" que busca aportar ideas sobre cómo hacer una mejor educación para una mejor sociedad (Adams y Bell, 2016; Agarwal-Rangnath, 2013; Au, Bigelow y Karp, 2007; Christensen, 2009; Cooke y Sweeney, 2017; Hytten, 2015; Lee, Menkart y Okazawa-Rey, 2007; Oakes y Lipton, 2007; entre otros).

Por otra parte, también resalta un enfoque de investigación que se preocupa por profundizar en las concepciones en educación. La idea que subyace es que las concepciones marcan las acciones, por lo que si queremos cambiar la educación es preciso, en primer lugar, conocer las concepciones para transformarlas y con ello modificar las acciones de los protagonistas del proceso educativo (Pozo, 2006; Remesal, 2011; Schraw y Olafson, 2003).

De esta forma, en esta investigación se busca profundizar en las concepciones que tienen los y las estudiantes en qué es una enseñanza socialmente justa mediante una aproximación fenomenográfica.

\section{Marco teórico}

No existe un acuerdo claro en definir una enseñanza socialmente justa. Así, por poner dos ejemplos recientes, Mauluci (2013) define la enseñanza socialmente justa como "una lucha continua por una escolarización más solidaria, equitativa y de apoyo en el aula (nivel micro), la escuela (nivel meso) y la comunidad/sociedad (nivel macro)" (p. 454). Sin embargo, Chubbuck (2010) considera que una enseñanza socialmente justa hace referencia al currículo y las pedagogías que brindan las mismas oportunidades de aprendizaje y logros para todos los alumnos y alumnas incluidos aquellos tradicionalmente excluidos. Para lograrlo, la investigadora considera que es necesario que los y las docentes identifiquen las causas estructurales de las desigualdades y determinen las herramientas necesarias para superar dichas inequidades.

Más allá de la definición concreta, en los últimos 20 años diversos investigadores e investigadoras de diferentes lugares del mundo han trabajado por comprender cómo es una enseñanza socialmente justa (Agarwal, Epstein, Oppenheim, Oyler y Sonu, 2010; Francis y Le Roux, 2011; North, 2015; Picower, 2012; Sensoy y DiAngelo, 2017; entre otros).

Agarwal, Epstein, Oppenheim, Oyler y Sonu (2010), por ejemplo, consideran que existen tres elementos claves en la enseñanza para la Justicia Social: a) planes de estudios que integran múltiples perspectivas, preguntas narrativas occidentales dominantes, y son incluyentes de la diversidad racial, étnica y lingüística, b) apoyo a los estudiantes a desarrollar una conciencia crítica de las injusticias que caracterizan a nuestra sociedad, y c) aportar a los estudiantes las herramientas necesarias para que ellos participen activamente en una democracia, a través del compromiso cívico y discusión deliberativa. De los planteamientos de Bigelow y otros (1994) y de Wade (2007) surgen las seis características que debe tener una enseñanza para la justicia social: centrada en el estudiante, colaborativa, experiencial, intelectual, crítica, multicultural y activista. 
Los postulados de Henry Giroux (1990) acerca del papel de profesorado en la consecución de una sociedad más justa siguen siendo un referente. Para este autor, el o la docente tienen siete funciones claves: a) fomentar una mentalidad crítica en los y las estudiantes, b) cuestionar el currículum y crear uno que favorezca el aprendizaje consciente y crítico de los estudiantes, c) analizar la política existente en la escuela, d) crear un discurso que favorezca la denuncia de las desigualdades y el cambio social, e) promover un proceso de enseñanza-aprendizaje a través de múltiples alfabetizaciones, es decir, que los estudiantes puedan aprender de diferente forma, f) combinar reflexión y acción en sus clases, convirtiendo a los estudiantes en agentes de cambio, g) lograr que los estudiantes interpreten críticamente el mundo y h) conseguir una educación para la acción transformadora. En esa línea, Hackman (2005) propone la necesidad que el o la docente posea cinco herramientas fundamentales para desarrollar una enseñanza socialmente justa: dominar el contenido a trabajar, promover el debate en el aula para desarrollar un pensamiento crítico en el alumnado, promover la acción de los estudiantes para el cambio social, favorecer la reflexión personal de los y las estudiantes y desarrollar una sensibilidad hacia la multiculturalidad. Para Sensoy y DiAngelo (2014, 2017) y Hytten (2015), enseñar para lograr una sociedad más justa supone a) conocerse más a uno mismo, a partir de procedimientos como la humildad reflexiva, tener la mente abierta a nuevas perspectivas, ampliar los límites del propio conocimiento y ser crítico con lo que te rodea, b) asumir que no todo es bueno ni malo, sino que existe la neutralidad y es necesario respetar todos los puntos de vista, c) reconocer la propia posición social para definir la forma en que cada uno piensa y actúa y d) ser empático, poniéndote en el lugar de todos aquello que te rodean.

Un paso más allá es la propuesta del investigador y activista estadounidense Bree Picower (2012), que concreta cómo debe ser una enseñanza socialmente justa en seis fases: Favorecer el autoconocimiento y mejorar la autoestima, Favorecer el respeto por los otros, Abordar aspectos sobre la injusticia social, Estudiar los movimientos sociales, Sensibilizar a la comunidad y Pasar a la Acción Social. Agarwal-Rangnath (2013), por su parte, propone cinco pasos también para construir una enseñanza socialmente justa: a) Inspirar sueños (referido a que lo que se enseñe en el aula tiene que conectar con los intereses, necesidades y realidad de los y las estudiantes), b) dibujar el panorama (centrado en comprender la realidad histórica y social desde múltiples perspectivas), c) aplicar lo conocido a distintas realidades históricas, c) conectar el pasado con el presente y d) facilitar el cambio (viendo las conexiones entre el pasado y el presente y siendo críticos con la realidad).

Existen pocos estudios empíricos que hayan profundizado en los elementos que conforman una enseñanza socialmente justa. Destaca la serie de investigaciones realizadas por Sharon Chubbuck $(2007,2008)$ relacionadas con la enseñanza socialmente justa. En 2008, realizó un estudio etnográfico para identificar las creencias docentes acerca de cómo es una enseñanza socialmente justa y cómo interaccionan estas creencias en la práctica docente. Los resultados muestran que las creencias se relacionan con el hecho de que la enseñanza socialmente justa incluya contenidos críticos del currículum, así como una práctica docente justa hacia los estudiantes. Además, si esta enseñanza no se aplica correctamente, no sirve para mejorar la realidad de las escuelas. Al comparar estas creencias con la práctica docente, se ha identificado que el querer ser socialmente justo normalmente hace que el docente tenga "miedo" a no hacerlo acertadamente y eso convierte muchas veces a los y las docentes en cómplices de las injusticias que se viven en las escuelas.

Otra investigación realizada por Chubbuck y Zembylas (2008) considera que estudiar las perspectivas emocionales puede facilitar la comprensión de la complejidad que supone llevar a cabo una enseñanza socialmente justa. Los hallazgos de este trabajo determinan, en primer lugar, que las emociones y su expresión desempeñan un papel importante y continuo en la enseñanza socialmente justa, y en segundo lugar, que la negociación emocional relacionada con la enseñanza socialmente justa puede proporcionar una comprensión más profunda del poder de cambio que tiene la escuela. Estos resultados son apoyados por el estudio llevado a cabo por Ramezanzadeh, Adel, Zareian y Ghazanfari (2017), quienes afirman que las emociones inciden directamente en la forma de desarrollar una enseñanza socialmente justa por parte del profesorado.

Especialmente interesante es el estudio de Kraft (2007), el cual realizó una investigación etnográfica con dos escuelas comprometidas socialmente para determinar cómo la enseñanza 
socialmente justa puede ayudar a entender las prácticas y políticas existentes en la escuela. Los resultados indican que es fundamental integrar los problemas de justicia en todo el currículo, utilizar prácticas de enseñanza socialmente justas y construir una comunidad escolar comprometida con las injusticias sociales.

Con esa base, el objetivo de esta investigación es determinar cómo es una enseñanza socialmente justa desde la perspectiva de los y las estudiantes de educación primaria y secundaria.

\section{Método}

La fenomenografía es el enfoque más adecuado para cumplir dicho objetivo dado que se centra en el conocimiento de las concepciones de los estudiantes, especialmente del proceso de enseñanza y aprendizaje (Åkerlind, 2012, 2017; Cossham, 2018; Collier-Reed, Ingerman y Berglund, 2009; González-Ugalde, 2014; Harris, 2008; Johnston y Salaz, 2017; Tight, 2016; Willis, 2017) y por ello es el que se sigue en esta investigación.

\subsection{Participantes}

Para garantizar un abanico de las formas cualitativamente distintas en las que las personas vivencian y entienden un determinado aspecto (Stamouli y Huggard, 2007) es fundamental tener claros los criterios para la selección de los y las participantes. En esta investigación se seleccionaron 32 estudiantes mediante un muestreo por cuotas con tres criterios de selección:

- Nivel educativo que cursan. Se seleccionaron 15 estudiantes de Educación Primaria y 17 de Educación Secundaria Obligatoria.

- Titularidad del centro. 17 de los estudiantes estudian en centros públicos y 15 en centros de titularidad privada (concertados y no concertados).

- Contexto socio-económico del centro educativo. Se ha seleccionado 14 estudiantes que asisten a centros situados en contextos favorables y 18 en contextos desafiantes. Para determinar el contexto de los centros educativos se tuvieron en cuenta tres criterios: a) el nivel socio cultural del barrio donde se encuentra el centro, b) el nivel socio-económico de las familias de los estudiantes que asisten a ese centro y c) las características sociales, culturales y lingüísticas de dichos estudiantes.

Las características de los y las participantes, de acuerdo con esos tres criterios se encuentra en el cuadro 1. Por cuestiones de accesibilidad, los y las estudiantes participantes en este estudio forman parte de centros escolares de la Comunidad de Madrid.

Cuadro 1. Distribución de los participantes en función del contexto, del nivel educativo y de la titularidad del centro

\begin{tabular}{lccccc}
\hline \multirow{2}{*}{ Contexto } & \multicolumn{2}{c}{ Primaria } & \multicolumn{2}{c}{ Secundaria } & \multirow{2}{*}{ Total } \\
\cline { 2 - 5 } & C. Públicos & C. Privados* & C. Públicos & C. Privados* & \\
\hline Favorable & 3 & 3 & 4 & 4 & 14 \\
Desafiante & 5 & 4 & 5 & 4 & 18 \\
\hline Total & 8 & 7 & 9 & 8 & 32 \\
\hline
\end{tabular}

(*) Tanto concertados como no concertados.

Fuente: Elaboración propia.

\subsection{Instrumento de obtención de información}

De acuerdo con el enfoque fenomenográfico utilizado, se ha empleado la entrevista fenomenográfica basada en el auto-relato (Trigwell, 2000). Las entrevistas fenomenográficas son conversaciones creativas que se construyen en colaboración entre el entrevistado y el entrevistador y en las que cobran especial relevancia las experiencias vividas por el entrevistado (Dortins, 2002) donde, en palabras de Marton (1996), "las experiencias y concepciones se construyen de forma 
conjunta entre el entrevistador y el entrevistado" (p. 4427). Siguiendo a Khan (2014) tuvimos en cuenta cuatro elementos fundamentales para realizar las entrevistas:

a) Se han conducido con un marco abierto y amigable que ha permitido a los y las estudiantes explorar su comprensión, experiencias o ideas de la manera más completa posible, creando un rapport adecuado para que se sintiesen cómodos, tranquilos y sin miedo a ser juzgados/as.

b) Se partió de la pregunta generadora de la entrevista: ¿qué es para ti una enseñanza socialmente justa? y se tuvo especial cuidado con las preguntas de seguimiento para poder profundizar con las concepciones de los y las participantes, pero sin sesgar la entrevista. El investigador no debe posicionarse frente a un determinado tipo de concepción, sino ser neutral y mostrar interés compartido con los participantes del estudio.

c) Se buscó explícitamente crear un entorno de conversación y confianza donde los y las estudiantes pudiesen reflejar completamente sus pensamientos.

\subsection{Trabajo de campo}

Las 32 entrevistas se desarrollaron entre febrero y mayo de 2016, con una duración de entre 15 y 20 minutos cada una. El proceso de selección de los y las participantes fue el siguiente:

1. Selección de los centros educativos más adecuados de acuerdo con los tres criterios de selección de los participantes anteriormente citados. Asimismo, se tuvo en cuenta que existiesen centros escolares de distinta titularidad, nivel educativo y ubicados en diferentes barrios de la Comunidad de Madrid.

2. Contacto con el equipo directivo de los centros educativos, enviándoles una carta con una presentación de la investigación y una explicación concreta del estudio a realizar.

3. Selección de los y las estudiantes junto con el equipo directivo. Una vez los directores y directoras tuvieron claros los objetivos y necesidades de nuestra investigación, estos realizaron una selección previa de estudiantes teniendo en cuenta que en primaria fuesen de cursos de quinto y sexto y en secundaria del segundo ciclo.

4. Envio de cartas a las familias a través del equipo directivo, explicando también de forma detallada la investigación a las familias, la participación de sus hijos/as, y solicitando un consentimiento firmado para que sus hijos/as participasen en la investigación y fuesen grabados y garantizando el anonimato y un tratamiento fiel de los datos obtenidos.

5. Selección final de los y las estudiantes participantes en el estudio, eligiendo 3 o 4 estudiantes de cada centro educativo (distribución final de los participantes en el cuadro 1).

Al finalizar, se transcribieron de forma completa y siguiendo de forma fiel las palabras de los y las estudiantes asignándoles una etiqueta con el siguiente formato [E1,pri,pu,des,p.1]. En él aparecen cinco informaciones: a) el número identificativo de cada estudiante, b) el nivel educativo que estudia (pri: educación primaria, y sec: educación secundaria), c) la titularidad del centro (pu: público, pr: privado), d) el contexto donde se ubica el centro (fav: favorable y des: desafiante), y e) la página de la transcripción donde se encuentra la cita.

\subsection{Análisis de datos}

Siguiendo un criterio de proximidad al discurso de los y las participantes y del enfoque fenomenográfico se ha llevado a cabo un análisis de datos en 6 fases para conocer las concepciones de los y las estudiantes (figura 1). 


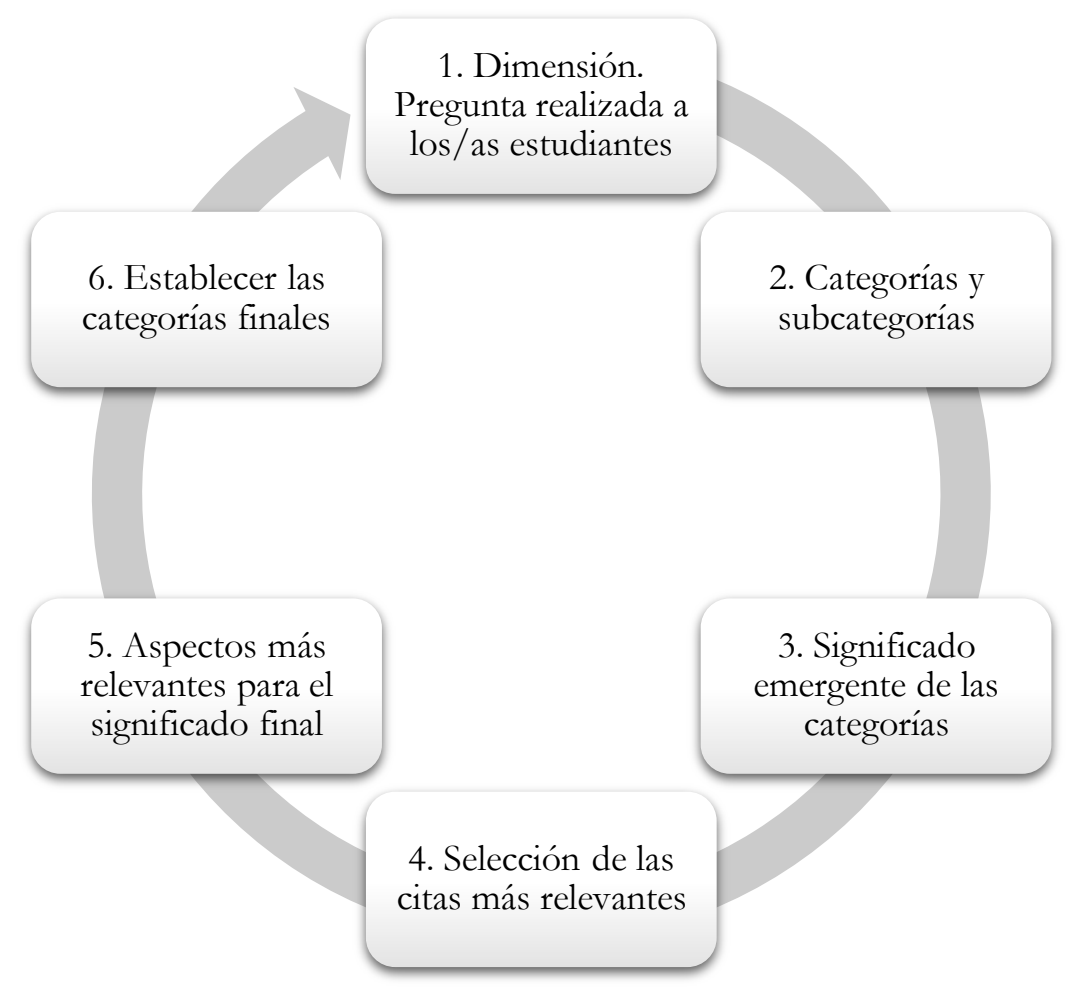

Figura 1. Pasos seguidos en el análisis del discurso de los estudiantes Fuente: Elaboración propia.

En primer lugar, se identificaron aquellas palabras y expresiones más utilizadas por los alumnos y alumnas referentes a qué conciben como una enseñanza socialmente justa. En segundo lugar, se organizaron las expresiones seleccionadas de las entrevistas para organizar las categorías iniciales de significado. Para analizar estas categorías iniciales, se utilizaron los tres principios propuestos por Sjostrom y Dahlgren (2002): Frecuencia, Posición y Pregnancy. En la tercera fase, se organizaron estas categorías en familias para dotarles de un significado conjunto y crear un árbol de significado de las concepciones de los y las estudiantes. Posteriormente, se seleccionaron las citas más representativas de cada una de las categorías establecidas, con especial énfasis en reflejar las ideas más relevantes de cada categoría. En la quinta fase, se compararon las citas seleccionadas con otras citas, creando un pool of meaning de todas las citas de los y las estudiantes, dando un sentido concreto y real al compararlas con otros fragmentos relevantes de las entrevistas realizadas acerca de cómo tiene que ser una enseñanza socialmente justa para los alumnos y alumnas (Harris, 2008). Por último, se determinaron las categorías finales y se eligieron las citas más idóneas para reflejar las palabras más interesantes de cada una de las categorías.

\section{Resultados}

Del proceso de investigación fenomenográfica, se han identificado cinco grandes categorías de concepciones, que pueden ser consideradas como elementos de lo que es una enseñanza socialmente justa para los y las estudiantes: aquélla que promueve un desarrollo integral de los alumnos y alumnas, la que se define por incluir contenidos que les haga conscientes y sensibles con las injusticias sociales, la centrada en promover la participación de los y las estudiantes en su aprendizaje y, por último, aquella que favorece que sean autónomos en su aprendizaje y que la evaluación les haga pensar y reflexionar, aprendiendo a ser críticos con lo que les rodea (figura 1). 


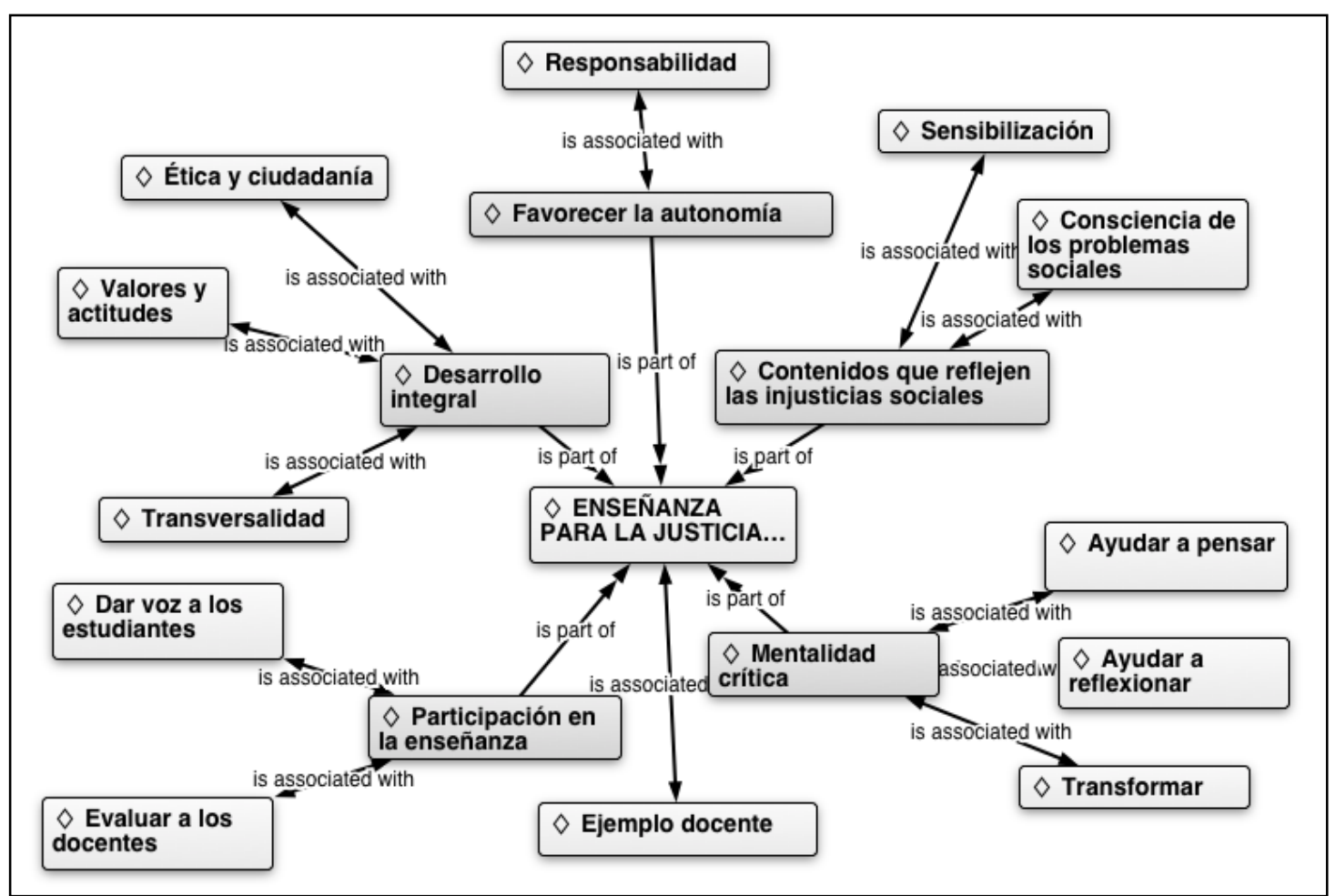

Figura 1. Concepciones de los estudiantes sobre la enseñanza para la Justicia Social

Fuente: Elaboración propia.

4.1. Educación y desarrollo integral de los estudiantes

La primera gran categoría encontrada es la concepción de los y las estudiantes sobre que si la enseñanza quiere transformar el mundo es fundamental que deje de centrarse únicamente en los aspectos cognitivos del aprendizaje, y promueva un aprendizaje que abarque también las destrezas, habilidades, valores y actitudes de los/las estudiantes. Los alumnos y alumnas consideran que la educación tiene el poder de prepararles para la vida, y esa preparación no puede lograrse si se enseñan sólo conocimientos:

En el instituto se dedican más a enseñarte contenidos que a formarte como persona y debería ser más equitativo [...] pidiendo la opinión de los alumnos de esos contenidos, que relación social tiene, que solución se podría buscar entre unos problemas u otros, como valoras tú esto. [E29,sec,pr,des,p.2]

Los profesores nos tendrían que enseñar, no solo centrarse en las teorías de las cosas, que nos expliquen las cosas de fuera, quépasa en el mundo, porque asi lo conoceríamos más, seriamos más conscientes y más críticos, y iríamos más preparados para el día de mañana. [E23,sec,pu,des,p.1]

Uno de los aspectos más señalados por los/las estudiantes es que los docentes trabajen y evalúen sus valores y actitudes, aspectos que consideran muy relevantes para su día a día. El alumnado considera que los contenidos de carácter teórico se olvidan fácilmente y no favorecen su desarrollo integral. Por ese motivo, defienden la necesidad de una enseñanza más globalizada que se preocupe de formarlos como personas:

Para cambiar el mundo el profesor nos tendría que enseñar valores para que no baya gente racista, maleducada y que vaya metiendo la gamba por todo el mundo. No todo es multiplicar o dividir o pasar de directo a indirecto, también es cómo te sientes tú, cómo se sienten los demás cuando les insultas. [E28,pri,pr,des,p.4] 
Potenciar asignaturas como ética o ciudadanía es una de las estrategias que destacan los estudiantes para favorecer el desarrollo de una mentalidad crítica, por un lado, y por otro que les ayude a formarse de manera integral como seres humanos. Los chicos y chicas entrevistados consideran que estas asignaturas deberían tener más peso en el currículum ya que son las que verdaderamente promueven el desarrollo de valores como el respeto, la tolerancia, la empatía o la humildad:

Hay una asignatura que es muy importante, la ética, que te enseña a ser persona; no es lo mismo entrar al colegio y ser un homófobo, un racista, un facha, a salir del instituto y gracias a th profesor o asignatura de ética te haya cambiado tu forma de ver el mundo; por ejemplo, no ves que los gays sean diferentes a ti, sino que tienes otros gustos, o que los negros o los chinos deben tener los mismos derechos que tú porque son igual que tú. [E9,sec,pu,des,p.2]

Se tendría que tener más en cuenta la ética, en lugar de centrarse tanto en matemáticas, lengua y eso, sería importante dar más peso a aquellas asignaturas que te ayudan a formarte como persona. [E8,sec,pri,fav,p.3]

\subsection{Contenidos sensibles a las injusticias sociales}

Para los/las estudiantes, una enseñanza para la justicia social es aquella que aborde de forma explícita y profundice en su conocimiento y comprensión. En este sentido, los contenidos que se trabajen en el aula tendrían que estar relacionados con temas actuales, que reflejen las injusticias que rodean a los/las estudiantes y a sus familias, ayudándoles a comprender por qué existen estas situaciones, así como que causas y qué consecuencias tienen. Conocer qué ocurre a su alrededor es un aspecto fundamental para los alumnos y alumnas entrevistados:

Es muy importante trabajar más los temas de los problemas sociales, no solo ver peliculas o hablar de cosas sin importancia, y eso nos ayuda a ser más conscientes, pero no solo en tutoría también por ejemplo en historia también se tendría que trabajar y que mirasen que no solo memorizásemos sino también que pensemos. [E22,sec,pu,des,p.2]

Si quieres cambiar lo actual primero tienes que conocerlo, para que no lo vuelvas a hacer mal, si no es un bucle. En historia por ejemplo, deberíamos trabajar más temas actuales, ligar lo pasado con lo actual, ver las relaciones, porque si a lo mejor tú te puedes saber mucho las fechas de la segunda guerra mundial pero luego no sabes ni por qué se produjo no sabes la economía de abora por qué es así, no sabes cómo funciona la política y eso.... Y las matemáticas lo mismo, las aplicaciones que tiene sobre la vida real. [E11 sec,pr,fav,p.3]

Mucha gente no es consciente de lo que pasa en el mundo, sí, lo ven en las noticias, pero hasta que no te pasa a ti, no te das cuenta de lo grave que puede llegar a ser, y los profesores nos tendrian que involucrar más en esos problemas, en esos temas y hacernos pensar de ello en los exámenes. [E10,sec,pu,fav,p.1]

Para conocer las situaciones injustas existentes, algunos estudiantes destacan la importancia de utilizar en el aula diferentes recursos didácticos como internet, periódicos, revistas, etc. Es conectar el aula con la vida.

Es muy interesante que nos hablen de los problemas del mundo, que usemos internet y veamos algún periódico, por ejemplo El País, y hablemos y debatamos sobre esa noticia. La mayoría de las personas viven el mundo chupi, la mayoría de los días hay comida, hay esto lo otro y no se dan cuenta de lo que pasa en el mundo. [E17,pri,pu,fav,p.2] 
Los profesores tendrian que enseñarnos lo que pasa en el mundo en el ordenador o leer textos de la violencia, los problemas y eso nos ayuda a pensar y en los exámenes nos podrían hacer pensar más preguntándonos de eso, poniendo nuestra opinión y lo que pienso en el examen. [E1,pri,pu,des,p.1]

Desde la perspectiva de los/las estudiantes, los y las docentes no solo tienen que tratar temas sobre las problemáticas existentes en el mundo sino especialmente sensibilizarles hacia ellas. Ponerse en la piel de otras personas que viven y sufren injusticias durante su día a día es fundamental para desarrollar habilidades como la empatía o el respeto. Los alumnos o alumnas conciben que sólo cuando llegan a ser sensibles con las problemáticas existentes verdaderamente son conscientes de ellas.

Nadie se da cuenta del mal que hace hasta que lo siente, y hay que hacer que de alguna manera la gente se ponga en el lugar de los que lo pasan mal; por ejemplo, que una persona que es homófoba que se ponga en el lugar del homosexual para que así pueda darse cuenta del daño que está haciendo; yo creo que es la única manera de que la gente cambie. Eso en la evaluación se puede trabajarponiéndonos casos prácticos y a ver cómo los resolveríamos, cómo actuaríamos, y eso nos ayudaría mucho a desarrollar la mente en el sentido práctico, en el sentido de la vida, porque no todo es matemáticas o filosofía, cuando salgamos de aqui nos vamos a encontrar con muchas otras cosas. [E7,sec,pr,fav,p.2]

4.3. Participación de los estudiantes en el proceso de aprendizaje

Otros estudiantes conciben que para una enseñanza socialmente justa es fundamental que se les convierta en protagonistas de su propio proceso de aprendizaje. Una estudiante de secundaria refleja la necesidad percibida por los estudiantes de convertirse en sujetos activos en el aula con las siguientes palabras:

Sería muy interesante que los profesores nos diesen voz, qué supiesen que queremos los alumnos. [E14,sec,pu,des,p.3]

Los muchachos y muchachas entrevistados coinciden en que se sienten meros consumidores pasivos de los conocimientos que se dan en el aula, considerando que si pudiesen participar más en su aprendizaje estarían más motivados e implicados. Participar, tanto en la enseñanza como en la evaluación, favorece que los estudiantes se responsabilicen más del proceso de enseñanzaaprendizaje:

Podría ayudar mucho que nos dejasen participar más en clase, porque nos motivaría más y nos implicariamos muchisimo más. Si días antes me preguntan ¿cómo podría hacer este examen?, pues me motivaría más, me implicaría y aparte lo conocería mucho mejor. [E29,sec,pr,des,p.2]

Algunos estudiantes también señalan que otro aspecto que convertirían la enseñanza en socialmente justa es que pudiesen evaluar a sus docentes. Si ellos son evaluados constantemente en el aula, ¿por qué no pueden valorar cómo trabajan sus profesores y profesoras? Esta es la pregunta que muchos de los estudiantes entrevistados se plantean. Los participantes consideran que, igual que sus docentes les indican mediante la evaluación qué y cómo tienen que cambiar para mejorar, ellos también deberían tener voz para explicarles a sus profesores y profesoras qué estrategias de enseñanza les ayudan más a aprender, qué trabajos son más provechosos o con qué tipo de evaluación se sienten más cómodos:

Me parecería bien que yo como alumna también puede evaluar a mi profesor; por ejemplo, mi padre es instructor y le evaluan, porque a veces para no echar a un profesor le dejan abi toda la vida y el profesor no hace nada, entonces a mi parece que no puedes pretender que el mundo cambie si a las 
personas que estás formando no les estás dando una persona que está funcionando; tendría que ser una evaluación mutua: del profesor a los alumnos y de los alumnos al profesor. [E11,sec,pri,fav,p.1]

4.4. Favorecer la autonomía a través de aprendizajes útiles para la vida

Algunos estudiantes resaltan la importancia que la enseñanza favorezca su autonomía personal. Conciben como fundamental priorizar aquellas habilidades que les ayuden a aprender y crecer de forma autónoma ya que va a favorecer que estos conocimientos puedan aplicarlos en su vida diaria. Así, los participantes valoran la utilidad de los aprendizajes y que tengan una transferibilidad a sus vidas:

Los profesores tienen que darte el sentimiento de que tienes que ser autónomo y tienes que saber moverte en el mundo, porque no sirve de nada muchas veces, te enseñan muchas cosas, que sípueden ser útiles para cuando vayas a la universidad, pero luego a lo mejor no enseñan otras cosas como ser persona, y entonces tienen que educar a personas no a trabajadores. [E13,sec,pri,fav,p.2]

Otro aspecto resaltado por los chicos y chicas entrevistados es que sus docentes sean un ejemplo a seguir. Así, los alumnos y alumnas explican que, si sus docentes son justos, reflexivos, críticos y llevan a cabo acciones contra las injusticias, ellos lo aprenderán. Consideran que los profesores y las profesoras tienen que ser un ejemplo a seguir ya que se ven reflejados en ellos. Para los estudiantes, los profesores son una pieza clave en el cambio social, ya que los consideran su referente:

Si mi profesor es crítico con el mundo, yo también lo seré, ser profesor es muy importante, porque educas a la gente joven. Según el profesor que tengas saldrán chicos bien educados o borregos. [E9,sec,pu,des, p.3]

\subsection{Enseñar a pensar y a ser críticos}

El último elemento fundamental para que la enseñanza pueda luchar contra las desigualdades existentes en la sociedad, según algunos estudiantes, es que ésta ayude a los estudiantes a pensar. Consideran que, en muchas ocasiones, la educación solamente persigue que sean capaces de reproducir o memorizar ciertos conocimientos cuando lo verdaderamente importante es que aquello que se les enseña les ayude a tener una mentalidad crítica. En palabras de dos estudiantes de secundaria entrevistadas:

Para cambiar el mundo es necesario que la evaluación te enseñe a aprender [...] a pensar, a juzgar, a ser crítico, a razonar. No se trabaja ni se evalúa casi nada la mentalidad crítica y es algo fundamental. [E22sec,pu,des, p.1]

Yo creo que nunca bay que obligar a alguien a hacer lo que no quiera, y cada uno piensa como le da la gana, porque eso es un derecho, pero eso si trata a la gente con respeto, enseñar a los colegios a tratar a los demás con respeto, sin molestar y cosas asi para que seamos mejores personas. [E15pri,pu,fav,p.2]

La escuela y los y las docentes tienen un papel fundamental en la generación de una conciencia crítica en los/las estudiantes. Para los chicos y chicas entrevistados, la escuela debería promover la realización de jornadas que conciencien a la comunidad sobre situaciones de injusticia, iniciativas como los estudiantes mentores o actividades transversales que favorezcan la reflexión y el compromiso social:

Por ejemplo, si se va a hacer algo en Fuenlabrada sobre el maltrato, que no sea algo puntual, sino más general, en Madrid y en España y que se impliquen a las escuelas, todo, no solo que a los niños pequeños se les diga no hay que pegar a las mujeres, sino que se hagan cosas también en secundaria y 


\begin{abstract}
en la universidad, y eso a nivel global, para que todos seamos conscientes y que nos implique, no solo que escuchemos una charla [...]. Hacer cosas puntuales en clase no sirve de nada, porque cuando suena el timbre, todo el mundo sigue igual, si dicen algo del racismo por ejemplo o esas cosas, y la gente está interesada, cuando se van a cada siguen igual y siguen siendo racistas con el vecino cbino. [E21,sec,pu,des,p.3]
\end{abstract}

Ser conscientes y críticos con las situaciones que rodean a los estudiantes favorece que no sean meros espectadores de los problemas, sino que actúen para cambiarlos. Desde la perspectiva de los estudiantes, ser críticos con la realidad supone reconocer su responsabilidad en las situaciones que se dan en su entorno y saber cómo actuar para transformarlas:

Los profesores deberían trabajar más la parte social para que te sientas involucrado en un futuro, no
ya solo ahora mismo, que sientas el peso de que es culpa tuya, sino que te sientas involucrado para
que el día de mañana digas esto no puede estar pasando y yo lo tengo que cambiar.
[E12,sec,pr,fav,p.1]

Nosotros tenemos que ser diferentes a lo que está pasando abora mismo en la sociedad, y nos tienen que transmitir las ganas y la motivación para trabajar más, esforzarnos más duro y poder hacer cosas, para cambiar la realidad. [E14,sec,pr,fav,p.2]

\title{
5. Discusión y conclusiones
}

Los resultados del estudio fenomenográfico, más que una serie de concepciones diferentes sobre lo que es una enseñanza socialmente justa, ha aportado una relación de elementos que, según los y las estudiantes, debe conformar dicha enseñanza. Así, han aparecido temas tales como la necesidad de una enseñanza centrada en su desarrollo como personas y en que éstos se conviertan en protagonistas y adquieran autonomía, un mayor grado de presencia y participación en todo el proceso de enseñanza-aprendizaje, que se trabaje en el aula temas relacionados con las injusticias y problemas de esta sociedad, favorecer su capacidad de reflexión y fomentar el sentido crítico tanto con lo que sucede en el aula como en su entorno.

Las concepciones que los/las estudiantes tienen sobre una enseñanza socialmente justa tienen muchas similitudes con, por ejemplo, los hallazgos del trabajo de Kraft (2007) en el que se estudian las prácticas de enseñanza justa de dos escuelas. En ambos casos se señalan elementos tales como la existencia de un currículum crítico que refleje la realidad e injusticias sociales que rodean al estudiante y que la enseñanza favorezca la participación del alumnado y de la comunidad educativa en el proceso de enseñanza-aprendizaje. También tiene puntos de conexión con algunas de las herramientas que señala Hackman (2005): desarrollar la capacidad reflexiva del alumnado para promover un pensamiento crítico, la participación y la acción de los estudiantes en la escuela y para el cambio social. Promover el pensamiento crítico y reflexivo, así como el énfasis en la empatía también es señalado en los estudios de Sensoy y DiAngelo (2014) y Hytten (2015).

En uno de sus estudios, Chubbuck (2007) señala la importancia de las creencias en la práctica educativa socialmente justa. Esta premisa también es concebida por los y las participantes de esta investigación, los cuales consideran que el profesorado refleja, con sus prácticas y actos, la forma que tiene de comprender la enseñanza, remarcando la necesidad de ser un ejemplo para sus estudiantes. Sin embargo, aspectos como la autonomía en el aprendizaje, trabajar por un desarrollo integral de los y las estudiantes son aspectos señalados por los y las estudiantes de este estudio fenomenográfico, que no hemos encontrado en otras investigaciones realizadas sobre la temática,quizá porque formen parte de una enseñanza de calidad, sin necesidad de tener ese enfoque socialmente justo.

Con todo ello, se puede concluir que las concepciones de los y las estudiantes sobre una enseñanza socialmente justa coinciden en gran medida tanto con las prácticas de las escuelas que trabajan con ese énfasis, como con los planteamientos de los y las estudiosos sobre el tema. Y ello lleva a pensar que una mayor participación de los y las estudiantes en las decisiones curriculares puede 
ayudar de una forma decisiva a la conformación de una enseñanza más centrada en la mejora de la sociedad. La participación estudiantil se erige, de esta forma, es una estrategia imprescindible en una educación de calidad socialmente comprometida (Belavi y Murillo, 2016).

Como toda investigación, este estudio tiene puntos fuertes, pero también aspectos a mejorar. Las principales fortalezas de esta investigación son, por un lado, el enfoque metodológico utilizado, ya que la fenomenografía es el enfoque más adecuado para estudiar las concepciones. La muestra de este estudio, tanto por cantidad (supera con claridad los 15-20 recomendados para este tipo de trabajos) como por su adecuada selección, que lleva a la saturación teórica de los resultados, es otro de los puntos fuertes del trabajo. También se destaca el enfoque de dar voz a los principales protagonistas del proceso educativo y conocer sus puntos de vista.

El carácter exploratorio de este trabajo, marcado en gran medida por la falta de estudios previos, es una limitación que podría ser superada mediante un estudio con una muestra más amplia. También sería importante conocer las concepciones de los otros protagonistas de la educación: el profesorado, las familias e incluso los tomadores de decisiones políticas.

Es necesaria otra educación que vaya más allá de mejorar el desempeño de los y las estudiantes en pruebas estandarizadas y dé pasos decisivos para contribuir a la construcción de un mundo mejor. Mandela decía que la educación es el arma más poderosa que podemos usar para cambiar el mundo, una enseñanza socialmente justa es una estrategia privilegiada para una educación que de verdad contribuya a la consecución de una sociedad más justa.

\section{Agradecimientos}

Información cegada para garantizar el anonimato.

\section{Referencias bibliográficas}

Adams, M. y Bell, L. A. (Eds.). (2016). Teaching for diversity and social justice. Londres: Routledge.

Agarwal-Rangnath, R. (2013). Social Studies, Literacy, and Social Justice in the Common Core Classroom. A guide for Teachers. Nueva York, NY: Teacher College Press.

Agarwal, R., Epstein, S., Oppenheim, R., Oyler, C. y Sonu, D. (2010). From ideal to practice and back again: Beginning teachers teaching for social justice. Journal of Teacher Education, 61(3), 237-

247. https://doi.org/10.1177/0022487109354521 http://journals.sagepub.com/doi/10.1177/0022487109354521

Akerlind, G. (2002, junio). Principles and Practice in Phenomenographic Research. Comunicación presentada en el Current Issues in Phenomenography Conference, Canberra, ACT.

Au, W., Bigelow, B. y Karp, S. (2007). Rethinking our classrooms: teaching for equity and justice. Social Studies Research and Practice, 4(2), 25-35.

Apple, M. W. (2012). Can education change society?. Londres: Routledge.

Belavi, G. y Murillo, F.J. (2016). Educación, democracia y justicia social. Revista Internacional de Educación para la Justicia Social, 5(1), 13-34. https://doi.org/10.15336/riejs2016.5.1 https://revistas.uam.es/riejs/article/view/4371

Christensen, L. (2009). Teaching for joy and justice: Re-imagining the language arts classrooms. Milwaukee: WI: Rethinking Schools.

Chubbuck, S. M. (2007). Socially just teaching and the complementarity of Ignatian pedagogy and critical pedagogy. Christian Higher Education, 6(3), 239-265. https://epublications.marquette.edu/edu_fac/327/

Chubbuck, S. M. (2008). A novice teacher's beliefs about socially just teaching: Dialogue of many voices. The New Educator, 4(4), 309-329. https://doi.org/10.1080/15476880802430254 https://www.tandfonline.com/doi/abs/10.1080/15476880802430254

Chubbuck, S. M. (2010). Individual and structural orientations in socially just teaching: Conceptualization, implementation, and collaborative effort. Journal of Techer Education, 61(3), 197-210. http://journals.sagepub.com/doi/10.1177/0022487109359777 
Chubbuck, S. M. y Zembylas, M. (2008). The emotional ambivalence of socially just teaching: A case study of a novice urban schoolteacher. American Educational Research Journal, 45(2), 274-318. https://www.jstor.org/stable/30069448?seq=1\#page scan tab contents

Collier-Reed, B. I., Ingerman, A. y Berglund, A. (2009). Reflections on trustworthiness in phenomenographic research: Recognising purpose, context and change in the process of research. Education as Change, 13(2), 339-355. https://doi.org/10.1080/16823200903234901 https://www.tandfonline.com/doi/abs/10.1080/16823200903234901

Cooke, N. A. y Sweeney, M. E. (Eds.). (2017). Teaching for justice: Implementing social justice in the LIS classroom. Nueva York, NY: Library Juice Press.

Cossham, A. F. (2018). An evaluation of phenomenography. Library and Information Research, 41(125), 17-31.

https:/ $/$ www.google.com/url?sa=t\&rct=j\&q=\&esrc=s\&source=web\&cd=2\&ved $=2 \mathrm{ahUK}$ EwiWjLvBz4XeAhWEzoUKHVMnC1MQFjABegQIBxAC\&url=https $\% 3 \mathrm{~A} \% 2 \mathrm{~F} \% 2 \mathrm{Fww}$ w. lirgjournal.org.uk $\% 2$ Findex.php $\% 2 \mathrm{Flir} \% 2 \mathrm{Farticle} \% 2 \mathrm{~F}$ download $\% 2 \mathrm{~F} 755 \% 2 \mathrm{~F} 769$ \&usg $=$ AOvVaw09GSzz987xCiOq8xFVhRBL

Dortins, E. (2002). Reflections on phenomenographic process: Interview, transcription and analysis. Quality Conversations: Research and Development in Higher Education, 25, 207-213.

https: / $/$ www.google.com/url?sa $=t \& r c t=j \& q=\& e s r c=s \& s o u r c e=w e b \& c d=1 \& c a d=r j a \& u a c$

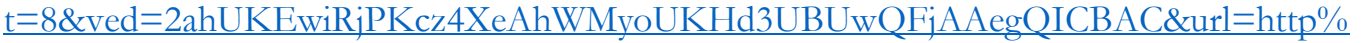
$3 \mathrm{~A} \% 2 \mathrm{~F} \% 2 \mathrm{Fciteseerx}$.ist.psu.edu $\% 2 \mathrm{Fviewdoc} \% 2 \mathrm{Fdownload} \% 3 \mathrm{Fdoi} \% 3 \mathrm{D} 10.1 .1 .90 .1182 \% 2$ 6rep \%3Drep1\%26type \%3Dpdf\&usg=AOvVaw1FoK64I3Uxd6TK12oInali

Francis, D. y Le Roux, A. (2011). Teaching for social justice education: the intersection between identity, critical agency, and social justice education. South African Journal of Education, 31(3), 299-311. https://www.ajol.info/index.php/saje/article/view/69813

Giroux, H. (1990). Los profesores como intelectuales. Barcelona: Paidós.

González-Ugalde, C. (2014). Investigación fenomenográfica. Magis, Revista Internacional de Investigación en Educación, 7(14), 141-158. https://doi.org/10.11144/Javeriana.M7-14.INFE

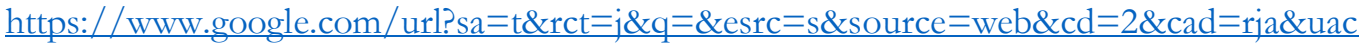
$\mathrm{t}=8 \& v e d=2$ ahUKEwjmqPKFz4XeAhVMyoUKHSBRD48QFjABegQICBAC\&url=http $\%$ 3A $\% 2 \mathrm{~F} \% 2$ Frevistas.javeriana.edu.co $\% 2$ Findex.php $\% 2 \mathrm{FMAGIS} \% 2 \mathrm{Farticle} \% 2 \mathrm{Fview} \% 2 \mathrm{~F} 1$ 1862\%2Fpdf\&usg=AOvVaw037OkaLQ4QVZp4YTzGqhA

Griffiths, M. (2003). Action for Social Justice in Education. Buckingham: Open University Press.

Hackman, H. W. (2005). Five essential components for social justice education. Equity \& Excellence in Education, 38(2), 103-109. https://doi.org/10.1080/10665680590935034 https://www.tandfonline.com/doi/abs/10.1080/10665680590935034?journalCode=ueee2 $\underline{0}$

Harris, L. R. (2008). A phenomenographic investigation of teacher conceptions of student engagement in learning. The Australian Educational Researcher, 35(1), 57-79. https://link.springer.com/article/10.1007/BF03216875

Hytten, K. (2015). Ethics in Teaching for Democracy and Social Justice. Democracy and Education, 23(2), 1-10. https://democracyeducationjournal.org/home/vol23/iss $2 / 1 /$

Johnston, N. y Salaz, A. M. (2017). Using phenomenography to bridge the gap between research and practice: a meta-analysis of three phenomenographic studies. Information Research, 22(4), 1-10. http://www.informationr.net/ir/22-4/rails/rails1614.html

Khan, S. H. (2014). Phenomenography: A qualitative research methodology in Bangladesh. International Journal on New Trends in Education and Their Implications, 5(2), 34-43. https://www.researchgate.net/publication/274642143 Phenomenography A qualitative research methodology in Bangladesh

Kraft, M. (2007). Toward a school-wide model of teaching for social justice: An examination of the best practices of two small public schools. Equity and Excellence in Education, 40(1), 77-86. https://www.tandfonline.com/doi/abs/10.1080/10665680601076601 
Kumashiro, K. K. (2015). Against common sense: Teaching and learning toward social justice. Londres: Routledge.

Lee, E., Menkart, D. y Okazawa-Retey, M. (Eds.). (2007). Beyond heroes and holidays: Practical guide to K12 anti-racist, multicultural educational and staff development. Washington, DC: Teaching for Change.

Marton, F. (1986). Phenomenography-a research approach to investigating different understandings of reality. Journal of Thought, 21(3), 28-49. https://www.jstor.org/stable/42589189

Murillo, F. J. y Hernández-Castilla, R. (2011). Trabajar por la justicia social desde la educación. REICE. Revista Iberoamericana sobre Calidad, Eficacia y Cambio en Educación, 9(4), 3-6.

https:/ $/$ www.google.com/url?sa $=t \& r c t=j \& q=\& e s r c=s \& s o u r c e=w e b \& c d=1 \& c a d=r j a \& u a c$ $\mathrm{t}=8 \& v e d=2$ ahUKEwjLooubzoXeAhUBXxoKHW1vB7IQFjAAegQICRAC\&url=http $\% 3$ A \%2F\%2Fwww.redalyc.org $\% 2 \mathrm{Fpdf} \% 2 \mathrm{~F} 551 \% 2 \mathrm{~F} 55122156001$.pdf\&usg=AOvVaw0dgDD Bg3j2P67z0SaZxG3H

Murillo, F.J. y Hernández-Castilla, R. (2014). Liderando escuelas justas para la justicia social. Revista Internacional de Educación para la Justicia Social, 3(2), 13-32.

https://revistas.uam.es/riejs/article/view/337

North, C. E. (2015). Teaching for social justice?: Voices from the front lines. Londres: Routledge.

Oakes, J. y Lipton, M. (2007). Teaching to change de world. Boston, MA: McGraw-Hill.

Oppenheim, R., Agarwal, R., Epstein, S., Oyler, C. y Sonu, D. (2009). From ideal to practice and back again: Beginning teachers teaching for social justice. Journal of Teacher Education, 61(3), 237247. http://journals.sagepub.com/doi/10.1177/0022487109354521

Picower, B. (2012). Practice what you teach: Social justice education in the classroom and the streets. Londres: Routledge.

Pozo, J. (2006). La cultura del aprendizaje en la sociedad del conocimiento. En J. Pozo, N. Scheuer, M. P. Pérez Echevarría, M. Mateos, E. Martín y M. De la Cruz (Eds.), Nuevas formas de pensar la enseñanza y el aprendizaje. Las concepciones de profesores y alumnos (pp. 29-53). Barcelona: Graó.

Ramezanzadeh, A., Adel, S. M. R., Zareian, G. y Ghazanfari, M. (2017). Emotions and socially just teaching: a qualitative study. Qualitative Research Journal, 17(2), 113-123. https://doi.org/10.1108/QRJ-11-2016-0072

https: / $/$ www.google.com/url?sa=t\&rct=j\&q $=\& e s r c=$ s\&source $=$ web\&cd $=1 \& c a d=$ rja\&uac $\mathrm{t}=8 \& v e d=2$ ahUKEwi4vtT9zYXeAhXkzIUKHdyGCQEQFjAAegQICBAC\&url=http $\% 3$ A $\% 2 \mathrm{~F} \% 2 \mathrm{Fprofdoc}$.um.ac.ir $\% 2 \mathrm{Farticles} \% 2 \mathrm{Fa} \% 2 \mathrm{~F} 1062513$.pdf\&usg $=\mathrm{AOvVaw0F}$ zXKEs AhsxRDPvqXyHo4

Remesal, A. (2011). Primary and secondary teachers' conceptions of assessment: A qualitative study. Teaching and Teacher Education, 27(2), 472-482. https://doi.org/10.1016/j.tate.2010.09.017 https://www.sciencedirect.com/science/article/pii/S0742051X1000168X

Schraw, G. y Olafson, L. (2003). Teachers' epistemological world views and educational practices. Journal of Cognitive Education and Psychology, 3(2), 178-235.

Sensoy, Ö. y DiAngelo, R. (2014). Respect differences? Challenging the common guidelines in social justice education. Democracy and Education, 22(2), 1-10.

https: / $/$ www.google.com/url?sa $=t \& r c t=j \& q=\& e s r c=s \&$ source=web\&cd=1\&cad=rja\&uac $\mathrm{t}=8 \& v e d=2$ ahUKEwi32MqzzYXeAhWJxYUKHVYQB58QFjAAegQICBAC\&url=https $\% 3 \mathrm{~A} \% 2 \mathrm{~F} \% 2 \mathrm{Fdemocracyeducationjournal.org} \% 2 \mathrm{Fcgi} \% 2 \mathrm{Fviewcontent}$.cgi $\% 3 \mathrm{Farticle} \% 3 \mathrm{D} 1$ 138\%26context $\% 3$ Dhome\&usg=AOvVaw36rt85ki7Qg0OW96r-pa6c

Sensoy, Ö. y DiAngelo, R. (2017). Is everyone really equal?: An introduction to key concepts in social justice education. Nueva Yorrk, NY: Teachers College Press.

Sjöström, B. y Dahlgren, L. O. (2002). Applying phenomenography in nursing research. Journal of Advanced Nursing, 40(3), 339-345. https://www.ncbi.nlm.nih.gov/pubmed/12383185

Stamouli, I. y Huggard, M. (2007). Phenomenography as a tool for understanding our students. En D. Brabazon y A. Ghani Olabi (Eds.), Actas del International Symposium for Engineering Education (pp. 181-186). Dublin: Dublin City University. 
Tight, M. (2016). Phenomenography: The development and application of an innovative research design in higher education research. International Journal of Social Research Methodology, 19(3), 319-338. https://doi.org/10.1080/13645579.2015.1010284 https://www.tandfonline.com/doi/abs/10.1080/13645579.2015.1010284?journalCode=ts $\underline{\mathrm{rm} 20}$

Trigwell, K. (2000). A phenomenographic interview on phenomenography. Phenomenography, 1, 6282.

Wade, R. (2007). Social studies for social justice: teaching strategies for the elementary classroom. Nueva York, NY: Teacher College Press.

Willis, A. S. (2017). The efficacy of phenomenography as a cross-cultural methodology for educational research. International Journal of Research \& Method in Education, 40, 1-17. https://doi.org/10.1080/1743727X.2017.1283398 https://www.tandfonline.com/doi/abs/10.1080/1743727X.2017.1283398?journalCode $=\mathrm{c}$ wse20

\section{Para citar este artículo}

Murillo, F.J. \& Hidalgo, N. (2018). Concepciones de Estudiantes sobre Enseñanza Socialmente Justa. Un Estudio Fenomenográfico. Revista Fuentes, 20(2), 75-89. [Fecha de consulta: $\mathrm{dd} / \mathrm{mm} / \mathrm{aa}]$.

doi: http://dx.doi.org/10.12795/revistafuentes.2017.v20.i2.05 